DISINTEGRINS

Peptides found in the venoms of various snakes that inhibit the function of integrins of the $\beta 1$ and $\beta 3$ classes.
Departments of ${ }^{\star}$ Oncology and ${ }^{\ddagger}$ Clinical Neurosciences, University of Calgary, 3330 Hospital Drive, Calgary, Alberta, Canada T3A 2Z1. ${ }^{\varsigma}$ School of Biological Sciences, University of East Anglia, Norwich NR4 7TJ, UK.

Correspondence to V.W.Y. e-mail:vyong@ucalgary.ca

\title{
METALLOPROTEINASES IN BIOLOGY AND PATHOLOGY OF THE NERVOUS SYSTEM
}

\author{
V. Wee Yong ${ }^{\star \ddagger}$, Christopher Power ${ }^{\sharp}$, Peter Forsyth ${ }^{\star}$ and Dylan R. Edwards ${ }^{\S}$ \\ Matrix metalloproteinases (MMPs) have been implicated in several diseases of the nervous \\ system. Here we review the evidence that supports this idea and discuss the possible \\ mechanisms of MMP action. We then consider some of the beneficial functions of MMPs during \\ neural development and speculate on their roles in repair after brain injury. We also introduce a \\ family of proteins known as ADAMs (a disintegrin and metalloproteinase), as some of the \\ properties previously ascribed to MMPs are possibly the result of ADAM activity.
}

Extracellular proteases are crucial regulators of cell function. The family of matrix metalloproteinases (MMPs) has classically been described in the context of extracellular matrix (ECM) remodelling, which occurs throughout life in diverse processes that range from tissue morphogenesis to wound healing. Recent evidence has implicated MMPs in the regulation of other functions, including survival, angiogenesis, inflammation and signalling. There are at least 25 members of the MMP family and, collectively, these proteases can degrade all constituents of the ECM. As a result of their potent proteolytic activity, abnormal MMP function can also lead to pathological conditions. The most widely studied disease that involves MMPs is cancer metastasis. In this case, the tumour cell is thought to use MMPs to overcome multiple structural barriers and establish a new focus of growth at a distant site from the primary tumour mass. In the nervous system, MMPs have also been associated with pathogenesis, particularly in multiple sclerosis (MS) and malignant gliomas ${ }^{1}$. A growing literature has linked MMPs to stroke, to Alzheimer's disease and to viral infections of the central nervous system (CNS). The goal of this review is to acquaint the reader with the biology of MMPs, particularly the functions of MMPs that are not associated with matrix turnover. We will highlight the roles of MMPs in pathology of the nervous system, and emphasize the fact that MMPs can also have physiological functions during CNS repair and ontogeny. Last, we will discuss the function of another group of metalloproteinases - ADAMs (a DISINTEGRIN and metalloproteinase) - in CNS pathophysiology, as these proteins might be responsible for many of the activities previously ascribed to MMPs.

\section{Metalloproteinases - the cast}

MMPs are part of a larger family of structurally related zinc-dependent metalloproteinases called metzincins. Other subfamilies of the metzincins are ADAMs, bacterial serralysins and the astacins. Metzincins use three histidine $(\mathrm{H})$ residues to bind the zinc ion at their active site. These residues occur in the conserved sequence motif HExxHxxGxxHZ, where $\mathrm{Z}$ is a family-specific residue - serine in most MMPs, aspartate in ADAMs, proline in serralysins and glutamate in astacins. In addition, there is a distinct $\beta$-turn at the active site, which is delineated by a methionine residue ('met-turn') and seems to be essential for activity. There is about 20\% similarity between metzincin subfamilies ${ }^{2}$, but identity at the catalytic domain is much higher.

On the basis of substrate preference and proteindomain considerations, MMP family members (TABLE 1) have been categorized into subgroups that include gelatinases, stromelysins, collagenases, membrane-type (MT)-MMPs and 'other MMPs'. However, there is much overlap in substrate specificity between subgroups. Structurally, MMPs are divided into three 
HEMOPEXIN

Serum glycoprotein that binds haem and transports it to the liver for breakdown and iron recovery. domains (FIG. 1): an amino-terminal propeptide region, an amino-terminal catalytic domain (which contains the zinc-binding motif) and a carboxy-terminal domain, which has a high level of similarity to members of the HEMOPEXIN family and is involved in ECM substrate binding for many MMPs. The carboxy-terminal hemopexinlike domain is a four-bladed $\beta$-propeller structure that is present in all MMPs except MMP7 and MMP26 (matrilysin and matrilysin-2). A hinge region, which is short in collagenases and long in other MMPs, connects the carboxy- and amino-terminal domains. A short signal sequence ('pre-domain') lies at the amino-terminal end of the protein, before the propeptide region, and is clipped off as newly synthesized MMPs travel to the cell surface. Last, unlike other MMP groups, the six MTMMPs are membrane proteins. MT4-MMP (MMP17) and MT6-MMP (MMP25) are glycosyl-phosphatidylinositol (GPI)-anchored MMPs (REF. 3), whereas the other MT-MMPs are transmembrane proteins.

The ADAM family of metalloproteinases is also referred to as MDC (metalloproteinase, disintegrin, cysteine-rich) proteins. Biological roles for ADAM members ${ }^{4}$ include specialized functions in cell adhe- sion, sperm-egg fusion (for example, ADAM1 and -2), myoblast fusion (ADAM12) and the ectodomain shedding of cell-surface proteins (for example, ADAM9, -10 and -17). Most ADAMs have a prodomain, a metalloprotease region, a disintegrin domain for adhesion, a cysteine-rich region, epidermal-growth-factor (EGF) repeats, a transmembrane module and a cytoplasmic tail (FIG. 1). So, ADAMs are unique among cell-surface proteins in having both adhesive and proteolytic activities. The cysteine-rich region and EGF repeats are thought to mediate cell fusion or the interaction of ADAMs with other molecules such as chaperone proteins. The cytoplasmic tails of ADAM9 and -12 interact with protein kinase $\mathrm{C}$ and with src, respectively, implicating some ADAM family members as signalling molecules $^{5,6}$. Although most ADAMs are transmembrane proteins, some members (for example, ADAM11, -12, -17 and -28) also have alternatively spliced forms that diverge before the transmembrane module to generate a soluble, secreted protein. It is important to note that only about half of the ADAM family members have a metalloproteinase domain that contains the catalyticsite consensus sequence, indicating that, in ADAMs

\begin{tabular}{|c|c|c|c|c|c|c|}
\hline Member & Name & $\begin{array}{l}M \text { latent/ } \\
\text { active (kDa) }\end{array}$ & $\begin{array}{l}\text { Furin } \\
\text { activation site }\end{array}$ & Collagen substrates & $\begin{array}{l}\text { Pro-MMP } \\
\text { substrates }\end{array}$ & Other matrix substrates* \\
\hline MMP1 & Collagenase 1 & $55 / 45$ & & I, II, III, VII, VIII, X & 2,9 & Agg, Gel, PG \\
\hline MMP2 & Gelatinase A & $72 / 66$ & & I, III, IV, V, VII, X, XI, XIV & $1,9,13$ & Agg, EL, FN, Gel, LN, PG, VN \\
\hline MMP3 & Stromelysin 1 & $57 / 45$ & & $\mathrm{III}, \mathrm{IV}, \mathrm{IX}, \mathrm{X}, \mathrm{XI}$ & $1,7,8,9,13$ & Agg, EL, FN, Gel, LN, PG, VN \\
\hline MMP7 & Matrilysin & $28 / 19$ & & IV, X & $1,2,9$ & Agg, Casein, EL, FN, Gel, LN, PG, VN \\
\hline MMP8 & Collagenase 2 & $75 / 58$ & & I, II, III, V, VII, VIII, X & & Agg, EL, FN, Gel, LN, \\
\hline MMP9 & Gelatinase B & $92 / 86$ & & $\mathrm{IV}, \mathrm{V}, \mathrm{VII}, \mathrm{X}, \mathrm{XIV}$ & & Agg, EL, FN, Gel, PG, VN \\
\hline MMP10 & Stromelysin 2 & $57 / 44$ & & III, IV, V, IX, X & 1,8 & Agg, EL, FN, Gel, LN, PG \\
\hline MMP11 & Stromelysin 3 & $51 / 44$ & Yes & & & \\
\hline MMP12 & Metalloelastase & $54 / 45 / 22^{\ddagger}$ & & IV & & Casein, EL, FN, Gel, LN, PG, VN \\
\hline MMP13 & Collagenase 3 & $60 / 48$ & & I, II, III, IV, VII, IX, X, XIV & 9 & Agg, FN, Gel \\
\hline MMP14 & MT1-MMP & $66 / 56$ & Yes & I, II, III & 2,13 & Agg, EL, FN, Gel, LN, \\
\hline MMP15 & MT2-MMP & $72 / 60$ & Yes & & 2 & Agg, FN, Gel, LN \\
\hline MMP16 & MT3-MMP & $64 / 52$ & Yes & III & 2 & Gel, FN \\
\hline MMP17 & MT4-MMP & $57 / 53$ & Yes & & & Fibrinogen/fibrin \\
\hline MMP18 & Collagenase 4 & $70 / 53$ & & I & & \\
\hline MMP19 & RASI1 & $54 / 45$ & & IV & & Gel, FN, LN \\
\hline MMP20 & Enamelysin & $54 / 22$ & & & & Amelogenin \\
\hline MMP21 & Xenopus MMP & $70 / 53$ & Yes & & & \\
\hline MMP22 & Chick embryo MMP & $51 / 42$ & & & & Casein, Gel \\
\hline MMP23 & & & Yes & & & \\
\hline MMP24 & MT5-MMP & & Yes & & 2 & Gel \\
\hline MMP25 & MT6-MMP & & Yes & IV & & Gel, FN \\
\hline MMP26 & Matrilysin 2/endometase & $28 / 19$ & & & & Gel \\
\hline MMP27 & Human MMP22§ & & & & & \\
\hline MMP28 & Epilysin & $56 / 45$ & Yes & & & Casein \\
\hline
\end{tabular}

MMP4, MMP5 and MMP6 were found to be identical to other MMPs, and are not listed according to this nomenclature. *The list of substrates, collated from REFS $15,56,100$, is by no means exhaustive and emphasizes those from the extracellular matrix. 期e activation of MMP12 involves the removal of the propeptide region to produce an intermediate active form (45 kDa), followed by atypical carboxy-terminal processing to produce the fully active enzyme (22 kDa). ${ }^{\S} \mathrm{MMP} 27$ is the human orthologue of chick embryo MMP22. M, molecular mass; MMP, matrix metalloproteinase; Agg, aggrecan; Gel, gelatin; PG, ptoteoglycan link protein; EL, elastin; FN, fibronectin; LN, laminin; VN, vitronectin; MT, membrane type; RAS I 1, rheumatoid arthritis synovial inflammation 1. 
MMPs
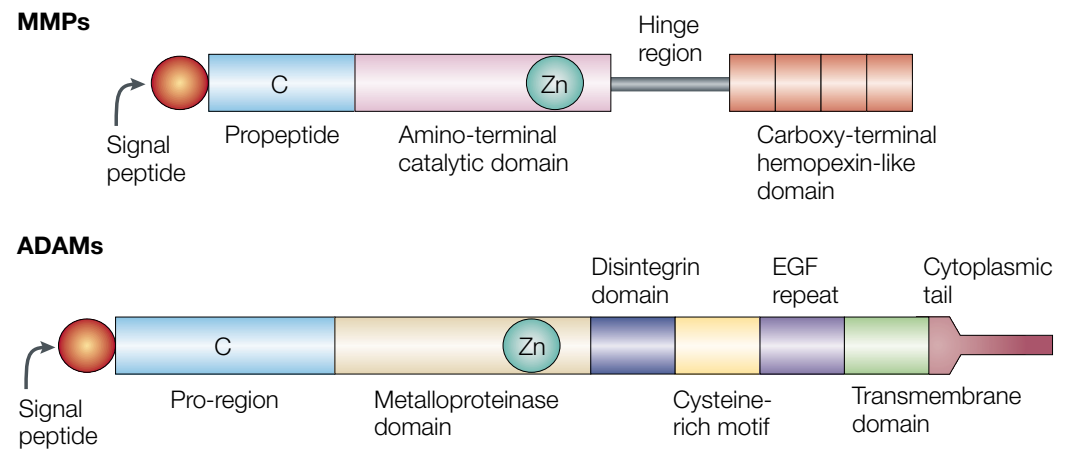

Figure 1 | Domain structure of MMPs and ADAMs. The generic structure of matrix metalloproteinases (MMPs) shown here describes most MMP family members. Note that gelatinases (MMP2 and -9) have a unique fibronectin type II-like domain inserted into the catalytic site, whereas MT-MMPs have a transmembrane domain at the carboxy terminus. Matrilysin (MMP7) lacks the hinge and the carboxyl terminus. The hemopexin-like module of MMPs contains four repeat units; the first and fourth are connected by a disulphide bridge. The ' $C$ ' at the propeptide region denotes the cysteine residue that ligates the zinc in the catalytic domain to keep the enzyme inactive. For ADAMs (a disintegrin and metalloproteinase), the core structure of most members is also depicted. Most ADAMs are integral membrane proteins owing to the presence of the transmembrane domain. However, alternative splicing generates a secreted form of some ADAMs. It should be noted that some ADAMs (ADAM2, -7, -11, -14, $18,-22$ and -29) lack the intact zinc-binding site and, furthermore, that the metalloprotease domain is not retained in several mature proteins of this family (for example, ADAM1 and -2) These proteins are therefore not considered true degradative enzymes. Last, the ADAMTS proteins are ADAMs that contain one or more thrombospondin type I motifs at the carboxyl terminus. They are also distinguished from ADAMs by the lack of epidermal-growth-factor (EGF)-like, transmembrane and cytoplasmic domains.

THROMBOSPONDIN

Homotrimeric glycoprotein found in platelets, and in the extracellular matrix of endothelial cells and fibroblasts. It is involved in platelet aggregation.

CHEMOKINES Small, secreted proteins that stimulate motile behaviour of leukocytes.

ZYMOGEN

Inactive precursor of a proteolytic enzyme that is synthesized and secreted in this form, and then activated by proteolytic cleavage.

FURIN

Endopeptidase with specificity for the consensus sequence Arg-X-Lys/Arg-Arg.

HYALURONAN Also known as hyaluronate. Polysaccharide of the glycosaminoglycans class highly abundant in the vitreous humour, the synovial fluid of joints and the umbilical cord. that lack metalloproteinase activity, this domain might fill another purpose, such as allowing protein-protein interactions. The expression of six ADAMs (ADAM9, $10,-12,-15,17$ and -19$)$ is widespread. By contrast, some ADAMs that are predicted to have metalloproteinase activity are expressed in a testis-specific fashion, and ADAM8 and -28 are predominantly haematopoietic. Recently, new members of the ADAM family have been identified ${ }^{7}$. They have THROMBOSPONDIN motifs and are therefore known as ADAMTSs. All ADAMTSs are secreted proteins. At present, at least 30 ADAMs and 10 ADAMTSs have been described.

The literature on the role of ADAMs in the CNS is still sparse. For example, an early description of ADAM10 in the CNS implicated this protein in oligodendrocyte and myelin biology ${ }^{8}$, although the molecule involved was not recognized as an ADAM until later. But despite the limited evidence, ADAMs might be important proteins in the nervous system. At least 17 ADAMs are found in the CNS (REF. 9) and, in fact, some of these are expressed predominantly in the brain (for example, ADAM22 and -23). Furthermore, many pharmacological studies on the role of MMPs have failed to discriminate between MMP or ADAM functions. In particular, many of the inhibitors based on the functional group hydroxamate, which chelate the zinc ion from the active site of MMPs, also have activity against ADAMs (REFS 10,11). Last, the thrombospondin motif of ADAMTSs confers degradative activity on several proteoglycans that are enriched in the CNS. Here, we will centre our discussion on two ADAMs, as several lines of evidence indicate their importance in the CNS: ADAM10 (kuzbanian) and ADAM17, also known as TACE (tumour-necrosisfactor- $\alpha$-converting enzyme).

\section{Regulation of metalloproteinase activity}

The activity of metalloproteinases is tightly regulated, as these molecules are potent proteolytic enzymes that are capable of widespread destruction. Their first regulatory step is at the level of transcription, as most MMPs are not constitutively expressed but are transcribed after cell activation. Transcription of many MMPs is promoted by inflammatory cytokines, growth factors, CHEMOKINES, oncogenes and cell-cell or cell-matrix interactions ${ }^{12}$.

Post-translational modifications provide a second level of MMP regulation. Many MMPs are expressed as inactive ZYMOGENS in which the cysteine residue at the propeptide region binds the zinc ion present at the catalytic site. Activating factors include the plasminogenplasmin cascade, as well as other MMPs (TABLE 1) that disrupt the interaction between cysteine and zinc (the so-called 'cysteine switch' mechanism) and then remove the propeptide region for full activation ${ }^{13}$. Non-proteolytic compounds such as sulphydryl-reactive agents (4aminophenylmercuric acetate) and denaturants (urea) can also activate zymogens. A subset of MMPs contains a cleavage site for FURIN-like prohormone convertases between the propeptide and catalytic domains; this subset includes the MT-MMPs, which are activated during secretion and appear on the cell surface in the active form.

A third means to control MMP activity is by the interaction of active MMPs with tissue inhibitors of metalloproteinases (TIMPs; see REFS 14,15 for comprehensive reviews). Four TIMPs are now known and they cause inactivation by binding to the catalytic site of MMPs. Interestingly, TIMPs are also required for the activation of some MMPs. In this regard, a complex formed by TIMP2 and the carboxyl terminus of proMMP2 has been found to bind MT1-MMP (MMP14) on the cell surface. An adjacent MT1-MMP molecule then removes the propeptide region of MMP2 (REF. 16). The pro-MMP-TIMP-MT-MMP trimolecular complex highlights another feature of MMP regulation: the active proteinase is found focally in the pericellular region, rather than being diffusely distributed. In addition, other means also exist to localize MMP activity to the pericellular region. For example, activated MMP2 can bind the $\alpha v \beta 3$ integrin, whereas active MMP9 can interact with the HYALURONAN receptor CD44 on the cell membrane ${ }^{17}$.

In the case of ADAMs, less is known about the regulation of their activity. Several ADAMs are kept in the inactive state through the interaction of a cysteine residue at the propeptide domain with zinc in the metalloproteinase module. So, as with MMPs, these ADAMs might be activated by the cysteine switch mechanism that disrupts the cysteine-zinc interaction to expose the catalytic site. ADAMs might also be regulated at the transcriptional level. For example, interleukin-1 (IL-1) can induce transcription of some ADAM members. Little is known of the physiological 


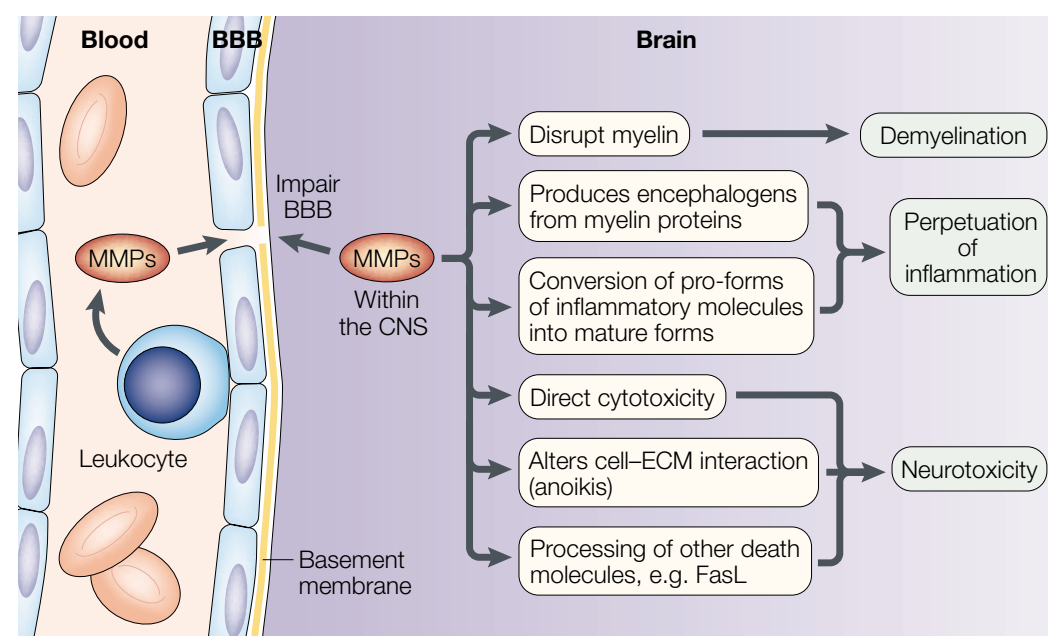

Figure 2 | Mechanisms by which MMPs contribute to neuropathology. Leukocytes express matrix metalloproteinases (MMPs) to facilitate their entry into the central nervous system (CNS). This process disrupts the basement membrane that surrounds the vasculature and results in blood-brain barrier (BBB) impairment. In the central nervous system, high MMP content and its indiscriminate localization result in perpetuation of an inflammatory response, which contributes to demyelination and neuronal or oligodendrocyte death. ECM, extracellular matrix.

RNASE PROTECTION ASSAY Technique for the quantitation of RNAs in solution. It is based on the use of a complementary radiolabelled probe that is hybridized to the target RNA after which the mixture is treated with RNase to degrade all remaining single-stranded RNA. The hybridized target will be protected from digestion and can be later visualized on a polyacrylamide gel.

GADOLINIUM-ENHANCED MAGNETIC RESONANCE IMAGING

Imaging technique in which gadolinium is introduced as a contrast agent, allowing for short data-acquisition times, large anatomical coverage and improved image quality. inhibitors of ADAMs. The crystal structure of the protease domain of human ADAM17 (TACE) shows that, although the active-site cleft is similar to what is found in MMPs, its secondary structure differs. These features might account for the finding that, whereas TIMP3 inhibits TACE, TIMP1 does not. Also, ADAM10 is inhibited by TIMP 1 and -3 , and by hydroxamates, but not by TIMP2 and -4 (REF. 18). So, TIMP3 might be a more general inhibitor of several ADAM family members, including the ADAMTSs. It is not known if more specific, endogenous non-TIMP ADAM inhibitors exist.

\section{Metalloproteinases and CNS diseases}

In the adult CNS, most MMPs are expressed at low or undetectable levels, although there are some exceptions. For example, RNASE PROTECTION ASSAYS have revealed a high constitutive expression of MMP11 and -14 in the adult mouse brain ${ }^{19}$. Similarly, the more sensitive polymerase chain reaction (PCR) technique has revealed the expression of MMP2, -3, -7, -9 and -13 in the normal rat spinal $\operatorname{cord}^{20}$. But, overall, MMPs are largely absent from the normal CNS and their upregulation has been reported in several neurological disorders and after injury. By contrast, in the case of ADAMs, over 17 family members are normally expressed in the adult CNS (REF. 9). For example, ADAM17 has been localized by immunohistochemistry to astrocytes and endothelial cells in adult human brain ${ }^{21}$, whereas ADAM8 has been detected in neurons and oligodendrocytes in the uninjured adult rat CNS (REF. 22). However, the literature on the expression of ADAMs in CNS pathology is limited.

We will divide our discussion of the role of MMPs and ADAMs in pathology into three parts: their role in neuroinflammation and MS, their involvement in malignant gliomas, and their participation in other neurological conditions such as stroke, viral infections and Alzheimer's disease.
Metalloproteinases in neuroinflammation and MS. MS is an immune disorder characterized by demyelination and axonal loss. The presence of proteinases in the cerebrospinal fluid (CSF) of patients with MS has been known for over 20 years; some of these proteinases were recently identified as MMPs. Specifically, MMP9, which is absent in the CSF of normal individuals, is upregulated in MS and in other inflammatory neurological diseases $^{23}$. MMP profiles in serum and in leukocytes are also altered in MS. So, when compared to healthy controls, MS patients show increased MMP9 messenger RNA in leukocytes and elevated MMP9 levels in serum. Similarly, the MMP9:TIMP1 ratio in the serum of people with MS is higher than normal ${ }^{24}$. The serum MMP9 content in MS patients is higher during relapse than in an off-period ${ }^{25}$. By using GADOLINIUM-ENHANCED MAGNETIC RESONANCE IMAGING (MRI) techniques, it was found that MS patients with high MMP9 and low TIMP1 levels tended to worsen ${ }^{26}$. Several groups have shown increased post-mortem expression of various MMP members (MMP2, -3, -7 and -9) in the brains of patients with MS (REFS 27,28). Cellular sources of MMPs in the diseased brain include infiltrating leukocytes (lymphocytes and macrophages) and intrinsic CNS cells (perivascular and parenchymal microglia, astrocytes and even neurons). Although it has been less thoroughly investigated, most studies indicate that TIMP1 and -2 might not be altered in MS compared with controls ${ }^{29}$. No data are available for TIMP3 and -4 .

MMPs are also dysregulated in experimental autoimmune encephalomyelitis (EAE), an animal model of MS. Increased expression of MMP7, -9 and -12 is observed in the rat CNS immediately preceding, and in parallel with, the development of symptoms. By contrast, transcripts encoding MMP2, $-3,-11$ and -13 were unchanged $^{20}$. Surprisingly, the elevation of MMP7 is not noted in mouse EAE, in which a prominent increase in MMP3, -9 and -12 occurs $^{30}$. So, overall, a common elevation of MMP9 and -12 is found in MS, and in mouse and rat EAE models.

The correlation between elevated MMPs and disease activity is probably causal, as inhibitors of metalloproteinase activity (for example, GM6001, Ro-9790 and BB1101) alleviate or prevent EAE (REFS 1,31). Also, 3-4-week-old mice that lack MMP9 are less impaired after the induction of EAE when compared with wildtype animals $s^{32}$. In humans, interferon- $\beta$, a drug used against MS, attenuates MMP9 production by T cells in vitro, in agreement with the decreased capacity of $\mathrm{T}$ cells to traverse ECM barriers ${ }^{33,34}$. In addition, MS patients treated with interferon- $\beta$ show a decrease in the content of serum MMP9 and a lowering of the number of MMP9-expressing leukocytes ${ }^{35}$. Together, these observations indicate that the antagonism of MMP function might contribute to the efficacy of interferon- $\beta$ in MS

Studies of MS and EAE have revealed several mechanisms that help to account for the involvement of MMPs in CNS pathology (FIG. 2). First, there is good evidence that $\mathrm{T}$ cells and other leukocytes use MMPs to penetrate ECM barriers, including the basement membrane that surrounds cerebral capillaries. In this regard, 
FAS LIGAND

Ligand for the Fas antigen, a transmembrane protein that mediates apoptosis and might be involved in negative selection of autoreactive $\mathrm{T}$ cells in the thymus.

GLIOBLASTOMA MULTIFORME Aggressive glioma, commonly of astrocytic origin, that infiltrates adjacent normal brain tissue. It accounts for about $10 \%$ of childhood brain tumours. the capacity of macrophages from MMP12-null mice to penetrate basement membranes is markedly diminished in vitro and in vivo ${ }^{36}$. Also, when T cells are applied onto a monolayer of endothelial cells that overlie a barrier of collagen matrix, the addition of GM6001, an metalloproteinase inhibitor, did not prevent the T cells from traversing the endothelial barrier. However, the entry of T cells into the collagen matrix is abolished ${ }^{37}$. One result of disrupting the basement membrane is the breakdown of the blood-brain barrier (BBB). This has been shown experimentally by the intracerebral injection of MMPs and the subsequent leakage of microvessels $^{31,38}$. In addition, there is a good correlation between gadolinium-enhanced MRI activity in humans, an index of BBB dysfunction, and the serum content of MMP9 (REFS 25,26).

MMPs have other undesirable consequences in the CNS parenchyma (FIG. 2). When injected into the CNS, MMPs can disrupt myelin and cause demyelination ${ }^{39}$. Furthermore, some of the fragments of the MMPmediated digestion of myelin basic protein (MBP) can induce EAE when injected into rodents ${ }^{31,40}$. Also, human MMP9 cleaves human MBP into peptide fragments, one of which is the immunodominant epitope in humans ${ }^{40}$. Another mechanism by which MMPs might promote an inflammatory response is by the conversion of precursor, inactive molecules into their activated forms. Tumour-necrosis factor (TNF)- $\alpha$ - a proinflammatory cytokine with several actions that include oligodendrocyte toxicity - is first made as a $26-\mathrm{kDa}$ membrane-associated protein that requires proteolytic conversion to the fully active $17-\mathrm{kDa}$ protein. Although TACE is known to be the protease that mediates the physiological maturation of TNF- $\alpha$ (REF. 41), it is clear that MMP7 and MT4-MMP can also efficiently mediate this conversion ${ }^{42}$. Other molecules, such as transforming growth factor (TGF)- $\alpha$, IL-6, TNF receptors, L-selectins and FAS LIGAND (FasL), are also synthesized as precursors that require processing by MMPs or ADAMs for maturation. So, in pathological conditions in which local levels of MMPs are high, these molecules might significantly modulate inflammation in the CNS.

Another pathogenic effect of MMPs in the CNS is neurotoxicity. Vos et al. ${ }^{43}$ reported that MMP1 is toxic to spinal cord neurons in vitro, and we found a similar neurotoxic effect of MMP2 (REF. 44). An alternative mechanism for MMP-mediated death might involve the phenomenon known as 'anoikis' (Greek for homelessness); cells that are attached to an ECM substrate survive through integrin signalling and death ensues when the cell is detached ${ }^{45}$. In principle, MMPs can indirectly cause cell death by degrading ECM and therefore interfering with cell attachment and integrin signalling. Indeed, neuronal death in the hippocampus after kainate-induced seizures in animals has been attributed to proteases that degrade laminin ${ }^{46}$. Finally, it has been noted that MMP7, but not MMP2, -3 or -9 , acts on cell-associated FasL to convert it into soluble FasL, which induces apoptosis of epithelial cells ${ }^{47}$. Conversely, MMP7-mediated cleavage of FasL protects sarcoma and colon carcinoma cells from chemotherapy-induced cytotoxicity. Given the presence of Fas and FasL in the CNS, MMPs could affect cell survival or death by similar mechanisms. In summary, the excessive production of MMPs in the CNS can be neurotoxic through several mechanisms (FIG. 2).

The literature on the upregulation of ADAMs in the CNS of MS patients is still in its infancy. Cells of the immune system produce various ADAMs (REF. 48), and it is likely that several ADAMs will be elevated in the CNS during neuroinflammation and MS. Also, with the exception of ADAM17 described above, much remains to be explored about the consequences of the aberrant expression of ADAMs in the CNS.

Metalloproteinases in malignant gliomas. An extensive literature links MMPs to tumour invasiveness and metastasis, as remodelling of the ECM is thought to be necessary for a tumour cell to advance. More recent studies have extended the roles of MMPs to other features of tumour progression, notably proliferation, angiogenesis and survival ${ }^{49}$. Essentially, all MMP members have been linked to cancers of various origins. So, it is not surprising that there are many reports of the increased expression of MMPs in brain tumours in situ and in vitro. Specifically, the upregulation of MMP2, -9 and all the MT-MMP members has been noted in highgrade specimens of GLIOBLASTOMA MULTIFORME (GBM) as compared to lower-grade cases or to non-transformed control brains ${ }^{50-52}$. The finding that AG3340 - an inhibitor of metalloproteinase activity - inhibits glioma growth, invasion and angiogenesis in animals ${ }^{53}$ highlights the importance of MMPs in the progression of brain tumours.

MMP2, MT1- and MT2-MMP have been localized to glioma cells in addition to CNS elements by in situ hybridization. By contrast, MMP9 is predominantly expressed in blood vessels at proliferative margin $\mathrm{s}^{51}$. A simplistic interpretation of these results is that MMP2 and MT-MMPs might regulate glioma invasiveness, whereas MMP9 might be crucial for angiogenesis. In this regard, the capacity of various glioma lines to migrate across a reconstituted basement membrane in vitro is correlated with the levels of MMP2 expression ${ }^{54}$. Furthermore, the ability of the C6 gliosarcoma line to migrate along myelin is related to MT1-MMP activity $^{55}$. As mentioned earlier, MT1-MMP facilitates the activation of MMP2, and an excessive MT1-MMP activity was recently noted in GBMs (REF. 52). Similarly, factors that stimulate glioma motility, such as hepatocyte growth factor/scatter factor, increase MMP2 or MT1-MMP levels. Conversely, inhibitors of MMP activity, including BB94 and BB2516, reduce glioma invasiveness in tissue culture.

Several MMPs can be activated by the serine protease plasmin ${ }^{56}$. The plasmin cascade starts with binding of urokinase plaminogen activator (uPA) to its receptor molecule $\mathrm{UPAR}$. This results in uPA activation and the subsequent conversion of plasminogen to plasmin by uPA. It is noteworthy that the uPA and uPAR are upregulated in GBMs (REF. 57), endowing the tumour with the machinery to activate its own MMPs 
effectively. In support of this idea, downregulation of uPAR in a glioma cell line led to decreased capacity to invade brain aggregates ${ }^{58}$. The expression of MMPs by glioma cells highlights a difference between CNS and systemic cancers. In the latter (for example, breast cancers), MMPs are often expressed by the stroma, rather than by the tumour itself. So, several mechanisms seem to exist for bestowing glioma cells with the capacity for autonomous, sustained growth.

TIMPs are also altered in gliomas. A decrease in TIMP2 content in GBM compared to lower-grade tumours has been noted in some $e^{52}$, but not all ${ }^{50}$, studies. This decrease would potentially remove a counterbalance for MMP activity. Although earlier studies noted a decrease of TIMP1 with increasing grades of glioma, later studies noted that it was actually upregulated in GBM (REFS 50,52). It is worth noting in this context that TIMPs have several properties that can contribute to the pathophysiology of cancer cells. Indeed, TIMPs have mitogenic action, and regulate survival and apoptosis independently of their inhibitory functions on MMPs (REF. 14).

Finally, elevated expression of various MMPs has been shown for other CNS tumour classes, including childhood astrocytomas, neuroblastomas and meningiomas. Overall, an excess of MMP activity seems to characterize many brain tumours. The development of metalloproteinase inhibitors to treat gliomas seems warranted and some are already being tested in clinical trials. A role for ADAMs in glioma biology remains to be defined and the related literature is limited at present ${ }^{59}$.

Metalloproteinases in other neurological conditions. A role for metalloproteinases in stroke is indicated by the finding that MMP2 and -9 are rapidly upregulated after focal cerebral ischaemia in rats ${ }^{60,61}$. In humans, elevation of brain MMP9 is detected post mortem within days of infarction and, interestingly, this protein remained elevated in patients that died months after the event ${ }^{62}$. In another report, MMP9 was strongly expressed by neutrophils in tissues from patients up to one week after an infarct, whereas the expression of MMP2 and -7 was less marked. From one week to five years, neutrophils were absent in the lesions and the large number of macrophages present were immunoreactive for MMP2 and -7 (REF. 28). The elevated MMP expression might contribute to the tissue destruction in stroke. As noted earlier, MMPs have the capacity to kill neurons (FIG. 2). Indeed, the intravenous treatment of rats with a neutralizing MMP9 antibody one hour before vessel occlusion reduced infarct size by $28 \%$ (REF. 61 ). In addition, the size of infarcts after ischaemia in MMP9-null mice was less than that observed in wild-type controls ${ }^{63}$.

Viral infections of the CNS have been increasingly associated with the production of MMPs. Elevated expression of MMP9 has been detected in the CSF of HIV-infected patients ${ }^{64}$. MMP2, -7 and -9 are increased in the CSF of patients with HIV-associated dementia compared to that of non-demented AIDS patients ${ }^{65}$. When brain-derived sequences of the HIV-transactivating protein TAT (tyrosine aminotransferase), obtained from demented patients, were expressed in U937 monocytoid cells and human macrophages, elevated MMP2 and -7 expression was obtained compared to TAT sequences from non-demented patients. These elevated MMPs resulted in neuronal death ${ }^{44}$. Collectively, the results indicate that HIV infection induces MMP expression in macrophages and that the latter are the possible sources of MMPs and neurotoxicity in the CNS of people with HIV-associated dementia. Other viruses, including EPSTEIN-BARR VIRUS, HTLV-1 and CORONAVIRUS ${ }^{66}$ have also been reported to increase the expression of MMPs in susceptible cell types. Of interest, these viruses have been associated with MS pathology, raising the possibility that viral-induced demyelination might involve MMP intermediaries.

MMPs are also associated with Alzheimer's disease, although the history has been full of false leads. Alzheimer's disease is characterized by plaques that are formed mostly by the deposition of amyloid- $\beta$ (A $\beta)$ a peptide derived from cleavage of the amyloid precursor protein (APP), an integral membrane molecule. Three proteases - $\alpha$-, $\beta$ - and $\gamma$-secretase - are involved in APP cleavage at different sites to generate $A \beta$ peptides of various length $s^{67}$. The predominant cleavage is mediated by $\alpha$-secretase, and this mode of cleavage is thought to be non-amyloidogenic. By contrast, the combination of $\beta$-secretase and $\gamma$-secretase activities releases the amyloidogenic $A \beta$ peptide. The aspartyl protease $B A C E$ ( $\beta$-site APP cleavage enzyme) is the best candidate to be the $\beta$-secretase ${ }^{68}$, whereas the $\gamma$-secretase activity depends on proteins known as presenilins.

It was initially suggested that MMP2 was the $\alpha$-secre$\operatorname{tase}^{69}$, but this idea was quickly disputed. Moreover, it was also suggested that MMP2 had $\beta$-secretase-like activity $^{70}$. Furthermore, the presence of immunoreactive TIMP in plaques of Alzheimer's disease led to the speculation that an MMP was excessively produced or activated in the plaques, as TIMPs have high affinity for MMPs and would therefore localize to sites of protease activity ${ }^{71}$. It was subsequently suggested that the $\alpha$-secretase activity was mediated by a non-MMP metalloproteinase, largely on the basis of studies using pharmacological inhibitors $^{72}$. Indeed, there is now good evidence for ADAM10 (REF. 73) and ADAM17 (REF. 74) as $\alpha$-secretases. If this proves to be the case, these ADAMs could have a protective action against Alzheimer's disease, as they would funnel APP towards the non-amyloidogenic pathway. It is worthwhile investigating whether the function of ADAMs is deficient or altered in Alzheimer's disease. Recently, hippocampal neurons that are immunoreactive for ADAM1 and - 2 were detected in Alzheimer's disease patients but not in age-matched controls ${ }^{75}$. In addition, levels of these proteins were also elevated ${ }^{75}$.

MMPs are also implicated in other diseases of the nervous system, including inflammatory myopathies ${ }^{76}$ and peripheral nerve axotomy. The involvement of MMPs in degenerative diseases of the CNS, including AMYOTROPHIC LATERAL SCLEROSIS, is an area of increasing interest. It is likely that the list of neurological disorders associated with aberrant MMP or ADAM expression will grow over the years. 


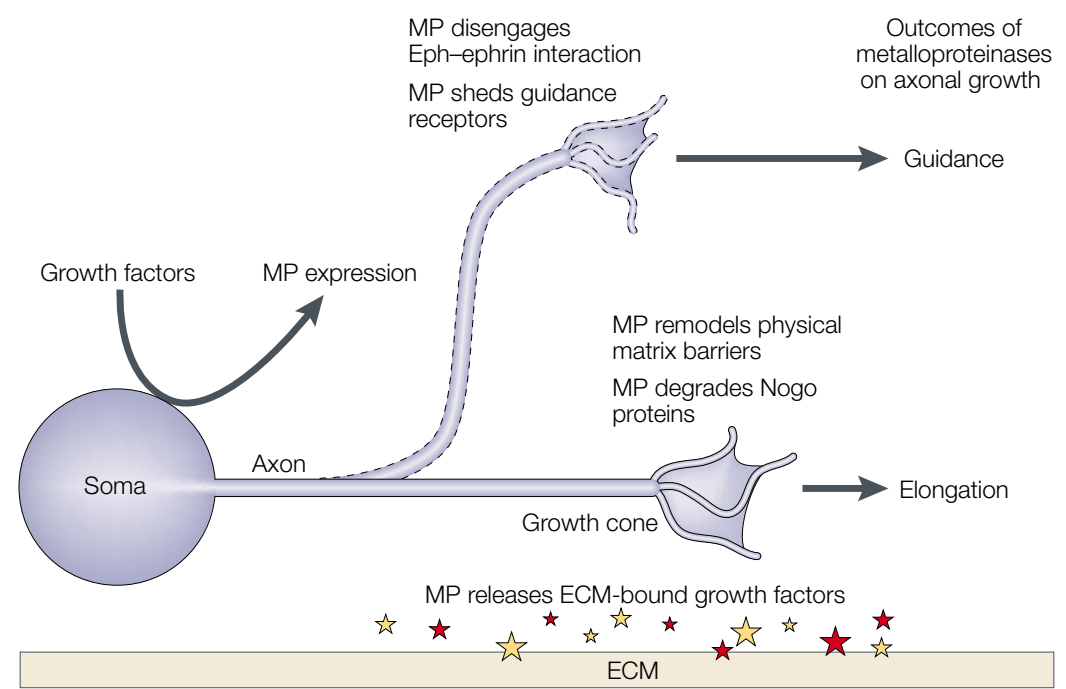

MP produces ECM matricryptin activity for neuronal survival and axonal growth

Figure 3 | Metalloproteinases regulate axonal growth. Several growth factors, including nerve growth factor (NGF), increase the expression of metalloproteinases (MPs) by neurons. MPs, in turn, regulate neurotrophic factor activity. One mechanism involves the release of growth factors that are anchored to the extracellular matrix (ECM). MPs that are located in the vicinity of growth cones can promote the elongation of axons owing to their ability to remodel the ECM and degrade inhibitory molecules such as the Nogo proteins. By interacting with molecules implicated in axonal guidance, for example, by shedding DCC (deleted in colorectal carcinoma) receptors or by breaking Eph receptor-ephrin bonds, MPs can affect the directional growth of axons.

CHONDROITIN SULPHATE PROTEOGLYCANS Important components of the extracellular matrix and connective tissue. These proteins contain hydrophilic, negatively charged polymers of glucuronic acid and sulphated $N$-acetyl glucosamine residues
Metalloproteinases in CNS ontogeny and repair

Although our discussion has so far revolved around the detrimental roles of metalloproteinases, it must be stressed that some of the functions of MMPs in the CNS might be beneficial. For example, some MMPs and TIMPs are expressed in the CNS during development ${ }^{77,78}$, pointing to their possible importance in brain maturation. Furthermore, MMPs are rapidly upregulated after nearly all types of injury to the CNS, including trauma ${ }^{19,79}$, indicating their possible relevance in tissue repair. In this section, we focus on the lesser-known beneficial aspects of metalloproteinases in the CNS.

In concordance with the classic role of MMPs in modulating the motility of cells across tissue matrices, metalloproteinases might regulate the migration of precursor cells to their destinations during neural development. Neural stem cells express MMP2 and all four TIMPs (REF. 80), and the migration of an oligodendrocyte progenitor requires MMP activity in vitro. The protein NOTCH affects cell-fate decisions in neurogenesis, and both ADAM10 (REF. 81,82) and ADAM17 (REF. 83) have been shown to activate the notch signalling cascade. Another role for MMPs in CNS development might lie in myelinogenesis, the process whereby oligodendrocytes extend several processes from their soma that reach and enwrap axons to form myelin. The initial expansion of oligodendroglial processes is immense and could require remodelling of the brain matrix by MMPs. This hypothesis has been tested and oligodendrocytes were found to express MMP9 during the period of myelinogenesis. Furthermore, the inhibition of MMP activity in vitro prevented the extension of oligo- dendroglial processes ${ }^{84}$. Other metalloproteinases could also regulate myelinogenesis, but this remains to be tested. The rabbit corpus callosum expresses MMP1 and -3 before and during myelination ${ }^{85}$, whereas ADAM10 is expressed in oligodendrocytes before and during myelinogenesis ${ }^{8}$.

In parallel with myelinogenesis, metalloproteinases also participate in axon elongation (FIG. 3). Early studies noted the presence of proteolytic activity at neuronal growth cones during attachment and reattachment events $^{86}$. Some of the activity is probably contributed by metalloproteinases, as interference with MMP activity inhibited growth-cone motility ${ }^{87}$. Inducers of neuronal differentiation and axonal outgrowth, such as nerve growth factor, laminin or retinoic acid, enhanced the expression of MMP2, -3 and -9 by dorsal root ganglion (DRG) neurons, PC12 and neuroblastoma cells ${ }^{88,89}$. Furthermore, growth cones of PC12 cells that stably expressed MMP3 had a reduced capacity to penetrate a reconstituted basement membrane. In vivo, Drosophila melanogaster flies that carry mutations in the protein Kuzbanian (the mammalian homologue is ADAM10) show axon stalling during development ${ }^{90}$. In a study in which neuritic outgrowth of DRG neurons that grow on top of normal adult nerves was evaluated, the slow neurite elongation was further reduced by treatment with metalloproteinase inhibitors ${ }^{91}$. By contrast, pretreating the nerves with recombinant MMP2 accelerated neurite growth ${ }^{91}$. Further studies led to the conclusion that DRG neurons expressed MMP2 that degraded and inactivated the neurite-inhibiting activity of $\mathrm{CHON}$ DROITIN SULPHATE PROTEOGLYCANS present on nerves, leading to the exposure of permissive laminin for neurite outgrowth ${ }^{91}$. Indeed, cleavage of a specific peptide bond in an ECM molecule leads to profound functional changes in other systems. For example, the cleavage of the Ala586-Leu 587 bond in the $\alpha 2$ chain of laminin- 5 by MMP2 induced migration of breast epithelial cells by exposing a cryptic pro-migratory site on laminin-5 (REF. 92). The biologically active sites in matrix molecules that become exposed after structural or conformational alterations have been called 'matricryptic sites', and the name matricryptins has been used to describe the resulting ECM fragments that have biological activity. Last, in concordance with the activity of MMP2 on proteoglycans described above, metalloproteinases might be used in the CNS to destroy other inhibitory proteins. C6 glioma cells and fibroblasts transfected with MT1MMP could digest NI250 (REF. 55), a Nogo protein identified as one of the most potent inhibitors of axonal elongation. In this way, some MMPs might act by neutralizing inhibitory proteins for axonal outgrowth.

Although these data implicate metalloproteinases in the creation of penetrable paths for axonal elongation (FIG. 3), metalloproteinases can also regulate guidance cues for growth cones. Ephrins are guidance molecules that bind to receptor tyrosine kinases of the Eph family. When the growth cone of a neuron that expresses Eph receptors encounters ephrin ligands on the surface of another cell, this facilitates the adherence of the cells to each other and bidirectional signalling to occur. The 


\begin{tabular}{l}
\hline Box $1 \mid$ Metalloproteinases in the CNS \\
\hline Beneficial activities \\
- Cell-fate specification \\
- Angiogenesis \\
- Myelinogenesis \\
- Survival of cells \\
- Axonal growth \\
- Signaling \\
- Repair processes \\
- Migration of neuronal precursors to final sites \\
- Terminate inflammation \\
Detrimental activities \\
- Tumorigenesis \\
- Disruption of blood-brain barrier \\
- Inflammation \\
- Demyelination \\
- Neuronal death \\
- Diseases, including glioma, stroke and \\
Alzheimers disease
\end{tabular}

growth cone then overcomes these adhesive forces and breaks away from the ephrin surface. Hattori et al. ${ }^{93}$ showed that the adhesive ephrin-Eph interaction is broken in vitro by ADAM10, which becomes activated after engagement of the Eph receptor. Another guidance molecule is netrin 1 , which binds a receptor known as DCC (deleted in colorectal carcinoma). When axon outgrowth from embryonic dorsal spinal explants was evaluated in vitro, the facilitatory activity of netrin 1 was potentiated by IC3 and GM6001, hydroxamate metalloproteinase inhibitors ${ }^{94}$. It was found that DCC was shed from the cell surface by the activity of an unidentified MMP; preventing the ectodomain shedding of DCC with a metalloproteinase inhibitor resulted in responsiveness to netrin 1 (REF. 94). The recent report of a phenotype-based GENE-TRAP screen to identify genes that control wiring patterns in the mouse CNS further implicates metalloproteinases in axonal guidance. ADAM23 was one of the genes identified in this screening; its inactivation in vivo led to neurological defects, tremor and ataxia ${ }^{95}$.

So, a beneficial role for metalloproteinases in neurogenesis and myelinogenesis during development seems to be supported by several lines of evidence. By contrast, the favourable functions (if any) of the MMPs that are upregulated in CNS disorders in adulthood are less clear. We have highlighted the detrimental effects of metalloproteinases in the injured adult CNS (FIG. 2) but some beneficial functions are also possible after injury, although they remain speculative at this point. What could they be? First, it is possible that metalloproteinases enable the migration of precursor cells to injured sites to replenish lost cells. A role in facilitating axonal regrowth, remyelination and angiogenesis is also possible on the basis of the functions of these proteases in development, as described earlier. Another possible role of MMPs in the adult CNS might involve their potential to affect signal transduction by, for example, linking G-protein-coupled-receptor signalling with the EGF pathway. In this context, the engagement of G-proteincoupled receptors has been shown to activate a membrane-associated metalloproteinase that converts proheparin-binding EGF to its mature form, triggering EGF signalling ${ }^{96}$. Regulation of synaptic plasticity could also be another function of metalloproteinases in CNS injury $^{77}$. Of interest, MMP1, $-2,-3$ and -9 were upregulated after spinal-cord transection in the AXоLOTL during the period of regeneration and subsided when recovery was near completion ${ }^{97}$.

MMPs could also participate in terminating inflammation in the CNS, in contrast to the perpetuation of inflammation that we discussed earlier (FIG. 2). The prolonged incubation of IL-1 $\beta$ with MMP3, and to a lesser extent MMP2 and -9, resulted in IL- $1 \beta$ degradation ${ }^{98}$. Furthermore, MMP2 binds to the chemokine MCP3 (macrophage chemoattractant protein 3) and cleaves its first four amino-terminal amino acids. The cleaved MCP3 binds to several chemokine receptors, acting as an antagonist $t^{99}$. So, MMPs expressed in the CNS might serve to abolish a chemotactic gradient for leukocyte entry. Interestingly, MMP9 has been reported to truncate the amino terminus of IL-8, leading to a 30 -fold potentiation of IL-8 activity ${ }^{100}$. Conversely, MMP9 degraded chemokines, such as gro- $\alpha$, leaving RANTES (also known as SCYA5) and MCP2 intact ${ }^{100}$.

In summary, metalloproteinases in the CNS have clear beneficial functions during development and might also have positive effects after injury. These effects contrast with the better-described detrimental roles of metalloproteinases in the CNS (BOX 1).

\section{The challenges ahead}

The challenges associated with defining the roles of MMPs in the CNS are several-fold. First, it is obvious that metalloproteinases can be both friends and foes in the CNS. We will therefore have to understand the balance between these states and the context that swings the pendulum between beneficial and detrimental roles. Second, a given metalloproteinase might have different properties at a given condition, dependent on factors such as spatial localization and cellular source. For example, MMP9 expressed by a macrophage in the vicinity of myelin might produce demyelination, whereas the same MMP elaborated by oligodendrocytes at the tip of their processes might promote remyelination. In this context, an inhibitor of MMP9 would simultaneously curb both detrimental and reparative processes. So, the challenge is to identify all the functions of a given metalloproteinase in a particular situation and to evaluate if the net result of its inhibition is worthwhile. A third challenge is related to establishing the identity of the metalloproteinase involved in a given function. Many of the pharmacological inhibitors that are available at present lack specificity towards members of a subfamily and will often antagonize the activity of members of different metalloproteinase families ${ }^{10,11}$. So, when functions are ascribed to particular metalloproteinases, 
it has to be clear, not only which metalloproteinase is involved, but also whether one is dealing with the right subfamily of metzincin metalloproteinases. Clearly, the next generation of pharmacological inhibitors must be able to discriminate between subfamilies and between members of each subfamily. Mice with null mutations for specific MMPs and ADAMs are already available and can help to discriminate functions of specific metalloproteinases. However, these valuable resources often show compensatory upregulation of activity of other MMPs as a result of the gene deletion and the analysis of these animals must therefore be interpreted with caution.

The identification of their physiological substrates is intimately related to the task of defining the functions of metalloproteinase $s$ in the CNS. Although it is clear that various MMP members can act on certain ECM components in vitro (TABLE 1), much remains to be discovered about their natural substrates in vivo. In the case of ADAMs, their CNS substrates are largely un known and research needs to focus on this area. Similarly, the ways in which metalloproteinases are transcribed and interact with other molecules, such as chemokines, remain to be elucidated.

Can any consensus be adopted at this point as to whether metalloproteinases should be targeted for inhi- bition to treat specific CNS diseases? Given the data that aberrant expression of MMPs is correlated with conditions such as MS, we suggest that the use of metalloproteinase inhibitors is warranted. If factors such as inflammation, which cause the disease to worsen, can be curbed, then the side effects of impairing reparative activities might be a reasonable trade-off. However, much more specific inhibitors and a clearer view of which metalloproteinase member to antagonize would help towards rational therapeutics.

In summary, much remains to be learned about the biology and pathology of metalloproteinases in the CNS. We welcome the explosion of this field, as the rewards of an increased understanding would seem to be immense.

\section{(2) Links}

DATABASE LINKS Multiple sclerosis | Alzheimer's disease | Hemopexin | MMP7 | MMP26 | MT4-MMP | MT6-MMP | ADAM1 | ADAM2 | ADAM12 | ADAM9 | ADAM10 | ADAM17 | src | ADAM11 | ADAM28 | ADAM15 | ADAM19 | ADAM8 | ADAM23 | TIMP2 | MT1-MMP | MMP9| TIMP3 | TIMP1 | TIMP4 | MMP11 | MMP14 | MMP3 | MMP13 | MMP12 | uPAR | TAT | NOTCH | Ephrins $\mid$ Netrin $1 \mid$ DCC $\mid$ MCP3 | RANTES
1. Yong, V. W., Krekoski, C. A., Forsyth, P. A., Bell, R. \& Edwards, D. R. Matrix metalloproteinases and diseases of the central nervous system. Trends Neurosci. 21, 75-80 (1998).

2. Stocker, W. et al. The metzincins - topological and sequential relations between the astacins, adamalysins, serralysins, and matrixins (collagenases) define a superfamily of zinc-peptidases. Protein Sci. 4, 823-840 (1995).

3. Kojima, S., Itoh, Y., Matsumoto, S., Masuho, Y. \& Seiki, M. Membrane-type 6 matrix metalloproteinase (MT6-MMP, MMP-25) is the second glycosyl-phosphatidyl inositol (GPI)anchored MMP. FEBS Lett. 480, 142-146 (2000).

4. Schlondorff, J. \& Blobel, C. P. Metalloprotease-disintegrins: modular proteins capable of promoting cell-cell interaction and triggering signals by protein-ectodomain shedding. J. Cell Sci. 112, 3603-3617 (1999) Excellent review that introduces the biology of ADAMs and then provides details on two members relevant to the CNS: ADAM10 and -17.

5. Izumi, Y. et al. A metalloprotease-disintegrin, MDC9/meltrin$\gamma / A D A M 9$ and PKC $\delta$ are involved in TPA-induced ectodomain shedding of membrane-anchored heparinbinding EGF-like growth factor. EMBO J. 17, 7260-7272 (1998)

6. Kang, Q., Cao, Y. \& Zolkiewska, A. Metalloprotease disintegrin ADAM 12 binds to the SH3 domain of Src and activates Src tyrosine kinase in $\mathrm{C} 2 \mathrm{C} 12$ cells. Biochem. $J$. 352, 883-892 (2000).

7. Kuno, K. et al. Molecular cloning of a gene encoding a new type of metalloproteinase-disintegrin family protein with thrombospondin motifs as an inflammation associated gene. J. Biol. Chem. 272, 556-562 (1997).

8. Chantry, A. Gregson, N. \& Glynn, P. Degradation of myelin basic protein by a membrane-associated metalloprotease: neural distribution of the enzyme. Neurochem. Res. 17 861-868 (1992).

9. Karkkainen, I., Rybnikova, E., Pelto-Huikko, M. \& Huovila, A P. Metalloprotease-disintegrin (ADAM) genes are widely and differentially expressed in the adult CNS. Mol. Cell. Neurosci 15, 547-560 (2000).

Key paper that describes the existence of several ADAMs in the CNS, although the functions of many of these members remain unknown.

10. Roghani, M. et al. Metalloprotease-disintegrin MDC9: intracellular maturation and catalytic activity. J. Biol. Chem. 274, 3531-3541 (1999)

11. Moss, M. L., White, J. M., Lambert, M. H. \& Andrews, R. C. TACE and other ADAM proteases as targets for drug discovery. Drug Discov. Today 6, 417-426 (2001).
12. Yong, V. W. The potential use of MMP inhibitors to treat CNS diseases. Exp. Opin. Invest. Drugs 8, 255-268 (1999).

13. Nagase, $\mathrm{H}$. Activation mechanisms of matrix metalloproteinases. Biol. Chem. 378, 151-160 (1997). This details the mechanisms by which several MMP members are activated.

14. Edwards, D. R. in Matrix Metalloproteinase Inhibitors in Cancer Therapy (eds Clendennin, N. J. \& Appelt, K.) 67-84 (Humana Press, Inc., Totawa, New Jersey, 2000).

15. Woessner, J. F \& Nagase, H. Matrix Metalloproteinases and TIMPS (Oxford Univ. Press, 2000)

16. Murphy, G. \& Knauper, V. Relating matrix metalloproteinase structure to function: why the 'hemopexin' domain? Matrix Biol. 15, 511-518 (1997)

17. Yu, Q. \& Stamenkovic, I. Localization of matrix metalloproteinase 9 to the cell surface provides a mechanism for CD44-mediated tumor invasion. Genes Dev. 13, 35-48 (1999).

18. Amour, A. et al. The in vitro activity of ADAM-10 is inhibited by TIMP-1 and TIMP-3. FEBS Lett. 473, 275-279 (2000).

19. Vecil, G. G. et al. Interleukin-1 is a key regulator of matrix metalloproteinase-9 expression in human neurons in culture and following mouse brain trauma in vivo. J. Neurosci. Res. 61, 212-224 (2000)

20. Clements, J. M. et al. Matrix metalloproteinase expression during experimental autoimmune encephalomyelitis and effects of a combined matrix metalloproteinase and tumor necrosis factor- $\alpha$ inhibitor. J. Neuroimmunol. 74, 85-94 (1997).

21. Goddard, D. R., Bunning, R. A. D. \& Woodroofe, M. N Astrocyte and endothelial cell expression of ADAM 17 (TACE) in adult human CNS. Glia 34, 267-271 (2001).

22. Schlomann, U., Rathke-Hartlieb, S., Yamamoto, S., Jockusch, H. \& Bartsch, J. W. Tumor necrosis factor $\alpha$ induces a metalloprotease-disintegrin, ADAM8 (CD156): implications for neuron-glia interactions during neurodegeneration. J. Neurosci. 20, 7964-7971 (2000).

23. Gijbels, K., Masure, S., Carton, H. \& Opdenakker, G. Gelatinase in the cerebrospinal fluid of patients with multiple sclerosis and other inflammatory neurological disorders. J. Neuroimmunol. 44, 29-34 (1992).

\section{This original manuscript describes the presence} This original manuscript describes the presence of

4. Lichtinghagen, R. et al. Expression of matrix

metalloproteinase-9 and its inhibitors in mononuclear blood
montix metalloproteinase- 9 and its inhibitors in mononuclear blood
cells of patients with multiple sclerosis. J. Neuroimmunol. 99, 19-26 (1999).
25. Lee, M. A. et al. Serum gelatinase B, TIMP-1 and TIMP-2 levels in multiple sclerosis. A longitudinal clinical and MR study. Brain 122, 191-197 (1999).

26. Waubant, E. et al. Serum MMP-9 and TIMP-1 levels are related to MRI activity in relapsing multiple sclerosis. Neurology 53, 1397-1401 (1999).

27. Cuzner, M. L. et al. The expression of tissue-type plasminogen activator, matrix metalloproteases and endogenous inhibitors in the central nervous system in endogenous inhibitors in the central nervous system in
multiple sclerosis: comparison of stages in lesion formation. multiple sclerosis: comparison of stages in lesion form
J. Neuropathol. Exp. Neurol. 55, 1194-1204 (1996).

28. Anthony, D. C. et al. Differential matrix metalloproteinase expression in cases of multiple sclerosis and stroke. Neuropathol. Appl. Neurobiol. 23, 406-415 (1997).

29. Mandler R. N. et al. Matrix metalloproteinases and tissue inhibitors of metalloproteinases in cerebrospinal fluid differ in multiple sclerosis and Devic's neuromyelitis optica. Brain 124, 493-498 (2001).

30. Pagenstecher, A., Stadler, A. K., Kincaid, A. L., Shapiro, S. D. \& Campbell, I. L. Differential expression of matrix metalloproteinase and tissue inhibitor of matrix metalloproteinase and tissue inhibitor of matrix
metalloproteinase genes in the mouse central nervous metalloproteinase genes in the mouse central nervous system in normal and in
152, 729-741 (1998).

31. Chandler, S. et al. Matrix metalloproteinases, tumor necrosis factor and multiple sclerosis: an overview. J. Neuroimmunol. 72, 155-161 (1997)

32. Dubois, B. et al. Resistance of young gelatinase B-deficient mice to experimental autoimmune encephalomyelitis and necrotizing tail lesions. J. Clin. Invest. 104, 1507-1515 (1999).

33. Stuve, $O$. et al. Interferon- $\beta$ decreases the migration of $T$ Iymphocytes in vitro: effects on matrix metalloproteinase-9. Ann. Neurol. 40, 853-863 (1996).

34. Leppert, D., Waubant, E., Burk, M. R., Oksenberg, J. R. \& Hauser, S. L. Interferon $\beta$ - $1 \mathrm{~b}$ inhibits gelatinase secretion and in vivo migration of human T cells: a possible mechanism for treatment efficacy in multiple sclerosis. Ann. Neurol. 40, 846-852 (1996). References 33 and 34 describe a key role of interferon- $\beta$ in MS: the alteration of MMP production by leukocytes.

35. Ozenci, V. et al. Multiple sclerosis: pro- and antiinflammatory cytokines and metalloproteinases are affected differentially by treatment with IFN- $\beta$. J. Neuroimmunol, 108, 236-243 (2000).

36. Shipley, J. M., Wesselschmidt, R. L., Kobayashi, D. K., Ley, T. J. \& Shapiro, S. D. Metalloelastase is required for 
macrophage-mediated proteolysis and matrix invasion in mice. Proc. Natl Acad. Sci. USA 93, 3942-3946 (1996).

37. Graesser, D. et al. The interrelationship of $\alpha 4$ integrin and matrix metalloproteinase-2 in the pathogenesis of experimental autoimmune encephalomyelitis. Lab. Invest. 78, 1445-1458 (1998)

38. Rosenberg, G. A. et al. TIMP-2 reduces proteolytic opening of blood-brain barrier by type IV collagenase. Brain Res. 576, 203-207 (1992).

39. Matyszak, M. K. \& Perry, V. H. Delayed-type hypersensitivity lesions in the central nervous system are prevented by inhibitors of matrix metalloproteinases. J. Neuroimmunol 69, 141-149 (1996).

40. Opdenakker, G. \& Van Damme, J. Cytokine-regulated proteases in autoimmune diseases. Immunol. Today $\mathbf{1 5}$ 103-107 (1994).

An excellent review that describes several key concepts on the generation of encephalogenic fragments after the incubation of myelin proteins with MMPs. The fact that one of these fragments is the immune trat of im

41. Black, R. A. et al. A metalloproteinase disintegrin that releases tumor-necrosis factor- $\alpha$ from cells. Nature $\mathbf{3 8 5}$, 729-733 (1997).

42. English, W. R. et al. Membrane type 4 matrix metalloproteinase (MMP17) has tumor necrosis factor- $\alpha$ convertase activity but does not activate pro-MMP2. J. Biol. Chem. 275, 14046-14055 (2000).

43. Vos, C. M. et al. Cytotoxicity by matrix metalloprotease- 1 in organotypic spinal cord and dissociated neuronal cultures. Exp. Neurol. 163, 324-330 (2000).

The toxicity of MMPs on CNS neurons was described for the first time in this article.

44. Johnson, J. B. et al. HIV-1 Tat neurotoxicity is prevented by matrix metalloproteinase inhibitors. Ann. Neurol. $\mathbf{4 9}$ 230-241 (2001).

45. Giancotti, F. G. \& Ruoslahti, E. Integrin signaling. Science 285, 1028-1032 (1999)

46. Chen, Z. L. \& Strickland, S. Neuronal death in the hippocampus is promoted by plasmin-catalysed degradation of laminin. Cell 91, 917-925 (1997).

47. Powell, W. C., Fingleton, B., Wilson, C. L., Boothby, M. \& Matrisian, L. M. The metalloproteinase matrilysin proteolytically generates active soluble Fas ligand and potentiates epithelial
1441-1447 (1999).

48. Yamamoto, S. et al. ADAM family proteins in the immune system. Immunol. Today 20, 278-284 (1999).

49. McCawley, L. J. \& Matrisian, L. M. Matri metalloproteinases: multifunctional contributors to tumor progression. Mol. Med. Today 6, 149-156 (2000).

50. Lampert, K. et al. Expression of matrix metalloproteinase and their tissue inhibitors in human brain tumors. Am. J. Pathol. 153, 429-437 (1998).

A thorough investigation into the expression of MMPs and TIMPs in malignant gliomas.

51. Forsyth, P. A. et al. Gelatinase-A (MMP-2), gelatinase-B (MMP-9) and membrane type matrix metalloproteinase-1 (MT1-MMP) are involved in different aspects of the pathophysiology of malignant gliomas. Br. J. Cancer 79, 1828-1835 (1999).

52. Nakada, M. et al. Roles of membrane type 1 matrix metalloproteinase and tissue inhibitor of metalloproteinases 2 in invasion and dissemination of human malignant glioma. J. Neurosurg. 94, 464-473 (2001).

53. Price, A. et al. Marked inhibition of tumor growth in a malignant glioma tumor model by a novel synthetic matrix metalloproteinase inhibitor AG3340. Clin. Cancer Res. $\mathbf{5}$, 845-854 (1999).

54. Uhm, J. H., Dooley, N. P., Villemure, J. G. \& Yong, V. W. Glioma invasion in vitro: regulation by matrix metalloprotease-2 and protein kinase C. Clin. Exp. Metastasis 14, 421-433 (1996)

55. Belien, A. T., Paganetti, P. A. \& Schwab, M. E. Membranetype 1 matrix metalloprotease (MT1-MMP) enables invasive migration of glioma cells in central nervous system white matter. J. Cell Biol. 144, 373-384 (1999).

56. Nagase, H. in Cancer Drug Discovery and Development: Matrix Metalloproteinase Inhibitors in Cancer Therapy (ed. Clendeninn, N. J. \& Appelt, K.) 39 (Humana Press, Inc., Totowa, New Jersey, 2000).

57. Yamamoto, M. et al. Expression and localization of urokinase-type plasminogen activator in human astrocytomas in vivo. Cancer Res. 54, 3656-3661 (1994).

58. Go, Y. et al. Inhibition of in vivo tumorigenicity and invasiveness of a human glioblastoma cell line transfected with antisense uPAR vectors. Clin. Exp. Metastasis 15, 440-446 (1997)

59. Harada, T. et al. The specific expression of three novel splice variant forms of human metalloprotease-like disintegrin-like cysteine-rich protein 2 gene in brain tissues and gliomas. Jpn. J. Cancer Res. 91, 1001-1006 (2000).

60. Rosenberg, G. A., Navratil, M., Barone, F. \& Feuerstein, G. Z. Proteolytic cascade enzymes increase in focal cerebral ischemia in rat. J. Cereb. Blood Flow Metab. 16, 360-366 (1996).

61. Romanic, A. M., White, R. F., Arleth, A. J., Ohlstein, E. H. \& Barone, F. C. Matrix metalloproteinase expression increases after cerebral focal ischemia in rats. Inhibition of matrix metalloproteinase-9 reduces infarct size. Stroke $\mathbf{2 9}$, 1020-1030 (1998)

62. Clark, A. W., Krekoski, C. A., Bou, S. S., Chapman, K. R. \& Edwards, D. R. Increased gelatinase A (MMP-2) and gelatinase B (MMP-9) activities in human brain after focal ischemia. Neurosci. Lett. 238, 53-58 (1997).

63. Asahi, M. et al. Role for matrix metalloproteinase 9 after focal cerebral ischemia: effects of gene knockout and enzyme inhibition with BB-94. J. Cereb. Blood Flow Metab. 20, 1681-1689 (2000).

64. Sporer, B. et al. Presence of matrix metalloproteinase-9 activity in the cerebrospinal fluid of human immunodeficienc virus-infected patients. J. Infect. Dis. 178, 854-857 (1998).

65. Conant, K. et al. Cerebrospinal fluid levels of MMP-2, 7, and 9 are elevated in association with human immunodeficiency virus dementia. Ann. Neurol. 46, 391-398 (1999).

66. Edwards, J. A., Denis, F. \& Talbot, P. J. Activation of glial cells by human coronavirus OC43 infection. J. Neuroimmunol. 108, 73-81 (2000).

67. Selkoe, D. J. The cell biology of $\beta$-amyloid precursor protein and presenilin in Alzheimer's disease. Trends Cell Biol. 8 447-453 (1998).

68. Vassar, R. et al. $\beta$-secretase cleavage of Alzheimer's amyloid precursor protein by the transmembrane aspartic proteas BACE. Science 286, 735-741 (1999)

69. Miyazaki, K., Hasegawa, M., Funahashi, K. \& Umeda, M. A metalloproteinase inhibitor domain in Alzheimer amyloid protein gelatinase $A$ possesses a $\beta$-secretase-like activity in cleaving the precursor. Nature 362, 839-841 (1993).

70. LePage, R. N. et al. Amyloid protein precursor of Alzheimer's disease. FEBS Lett. 377, 267-270 (1995).

71. Peress, N., Perillo, E. \& Zucker, S. Localization of tissue inhibitor of matrix metalloproteinases in Alzheimer's disease and normal brain. J. Neuropathol. Exp. Neurol. 54, 16-22 (1995).

72. Qiu, W. Q., Ye, Z., Kholodenko, D., Seubert, P. \& Selkoe, D. J. Degradation of amyloid $\beta$-protein by a metalloproteinase secreted by microglia and other neural and non-neural cells. J. Biol. Chem. 272, 6641-6646 (1997).

73. Lammich, S. et al. Constitutive and regulated $\alpha$-secretase cleavage of Alzheimer's amyloid precursor protein by a disintegrin metalloprotease. Proc. Natl Acad. Sci. USA 96 3922-3927 (1999).

74. Buxbaum, J. D. et al. Evidence that tumor necrosis factor $\alpha$ converting enzyme is involved in regulated $\alpha$-secretase cleavage of the Alzheimer amyloid protein precursor. J. Biol. Chem. 273, 27765-27767 (1998).

75. Gerst, J. L. et al. Altered cell-matrix associated ADAM proteins in Alzheimer disease. J. Neurosci. Res. 59, 680-684 (2000)

76. Kieseier, B. C. et al. Expression of specific matrix metalloproteinases in inflammatory myopathies. Brain $\mathbf{1 2 4}$ 341-351 (2001)

77. Vaillant, C., Didier-Bazes, M., Hutter, A., Belin, M. F. \& Thomas, N. Spatiotemporal expression patterns of metalloproteinases and their inhibitors in the postnat developing rat cerebellum. J. Neurosci. 19, 4994-5004 developing
(1999).

78. Fager, N. \& Jaworski, D. M. Differential spatial distribution and temporal regulation of tissue inhibitor of metalloproteinase mRNA expression during rat central nervous system development. Mech. Dev. 98, 105-109 (2000).

79. Xu, J. et al. Glucocorticoid receptor-mediated suppression of activator protein- 1 activation and matrix metalloproteinase expression after spinal cord injury. J. Neurosci. 21, 92-97 (2001).

80. Frolichsthal-Schoeller, P. et al. Expression and modulation of matrix metalloproteinase-2 and tissue inhibitors of metalloproteinases in human embryonic CNS stem cells. Neuroreport 10, 345-351 (1999).

81. Pan, D. \& Rubin, G. M. Kuzbanian controls proteolytic processing of Notch and mediates lateral inhibition during Drosophila and vertebrate neurogenesis. Cell 90, 271-280 (1997).
82. Qi, H. et al. Processing of the notch ligand delta by the metalloprotease Kuzbanian. Science 283, 91-94 (1999).

83. Brou, B. et al. A novel proteolytic cleavage involved in Notc signaling: the role of the disintegrin-metalloprotease TACE. Mol. Cell 5, 207-216 (2000).

84. Oh, L. Y. S. et al. Gelatinase B/matrix metalloproteinase- 9 is required for oligodendroglial process extension in vivo and in vitro. J. Neurosci. 19, 8464-8475 (1999).

85. Del Bigio, M. R. \& Jacque, C. M. Localization of proteinase expression in the developing rabbit brain. Brain Res. Dev. Brain Res. 86, 345-347 (1995).

86. Pittman, R. N. Release of plasminogen activator and a calcium-dependent metalloproteinase from cultured sympathetic and sensory neurons. Dev. Biol. 110, 91-101 (1985)

87. Sheffield, J. B., Krasnopolsky, V. \& Dehlinger, E. Inhibition of retinal growth cone activity by specific metalloproteinase inhibitors in vitro. Dev. Dyn. 200, 79-88 (1994).

88. Machida, C. M., Rodland, K. D., Matrisian, L., Magun, B. E. \& Ciment, G. NGF induction of the gene encoding the protease transin accompanies neuronal differentiation in PC12 cells. Neuron 2, 1587-1596 (1989).

89. Chambaut-Guerin, A. M., Herigault, S., Rouet-Benzineb, P. Roucher, C. \& Lafuma, C. Induction of matrix metalloproteinase MMP-9 (92-kDa gelatinase) by retinoic acid in human neuroblastoma SKNBE cells: relevance to neuronal differentiation. J. Neurochem. 74, 506-517 (2000).

90. Fambrough, D., Pan, D., Rubin, G. M. \& Goodman, C. S. The cell surface metalloproteinase/disintegrin Kuzbanian is required for axonal extension in Drosophila. Proc. Natl Acad. Sci. USA 93, 13233-13238 (1996).

91. Zuo, J., Ferguson, T. A., Hernandez, Y. J., Stetler-Stevenson, W. G. \& Muir, D. Neuronal matrix metalloproteinase-2 degrades and inactivates a neurite-inhibiting chondroitin sulfate proteoglycan. J. Neurosci. 18, 5203-5211 (1998) This very elegantly conducted project shows the expression of MMP2 by neurons and the MMP-2mediated degradation of inhibitory ECM molecules in peripheral nerves, which exposes permissive substrates for axonal elongation.

92. Giannelli, G., Falk-Marzillier, J., Schiraldi, O., StetlerStevenson, W. G. \& Quaranta, V. Induction of cell migration by matrix metalloprotease-2 cleavage of laminin-5. Science 277, 225-228 (1997).

93. Hattori, M., Osterfield, M. \& Flanagan, J. G. Regulated cleavage of a contact-mediated axon repellent. Science 289, 1360-1365 (2000).

94. Galko, M. J. \& Tessier-Levigne, M. Function of an axonal chemoattractant modulated by metalloproteinase activity. Science 289, 1365-1367 (2000).

95. Leighton, P. A. et al. Defining brain wiring patterns and mechanisms through gene trapping in mice. Nature $\mathbf{4 1 0}$ 174-179 (2001).

96. Prenzel, N. et al. EGF receptor transactivation by G-protein coupled receptors requires metalloproteinase cleavage of proHB-EGF. Nature 30, 884-888 (1999).

97. Chernoff, E. A. G., O'Hara, C. M., Bauerle, D. \& Bowling, M. Matrix metalloproteinase production in regenerating axolotl spinal cord. Wound Repair Regen. 8, 282-291 (2000)

98. Ito, A. et al. Degradation of interleukin- $1 \beta$ by matrix metalloproteinases. J. Biol. Chem. 271, 14657-14660 (1996).

99. McQuibban, G. A. et al. Inflammation dampened by gelatinase A cleavage of monocyte chemoattractan protein-3. Science 289, 1202-1206 (2000).

A novel role for MMPs was revealed by this report. The interation of MMP2 with a chemy this report. A chemokine receptors, thereby abolishing a directional cue for the migration of leukocytes.

100. Van den Steen, P. E., Proost, P., Wuyts, A., Van Damme, J. \& Opdenakker, G. Neutrophil gelatinase B potentiates interleukin-8 tenfold by aminoterminal processing, whereas it degrades CTAP-III, PF- 4 and gro- $\alpha$ and leaves RANTES and MCP-2 intact. Blood 96, 2673-2681 (2000).

\section{Acknowledgements}

We thank the Canadian Institutes for Health Research and the Multiple Sclerosis Society of Canada for operating funds. V.W.Y. acknowledges the past and present trainees in his laboratory who have contributed to his understanding of metalloproteinases in the CNS: V. Brundula, S. Chabot, N. Dooley, J. Dowden, F. Giuliani, U. Ladiwala, P. Larsen, D. M. Le, L. Y. S. Oh, O. Stuve, J. H. Uhm, G. Vecil, C. Verhaghe and Y. Zhou. Because of space limitations, important contributions by several authors could not be included. We apologize for this omission. 
Multiple sclerosis

http://www.ncbi.nlm.nih.gov/entrez/dispomim.cgi?id=126200

Alzheimer's disease

http://www.ncbi.nlm.nih.gov/entrez/dispomim.cgi?id=104300

hemopexin

http://www.ncbi.nlm.nih.gov/LocusLink/LocRpt.cgi?l=3263

MMP7

http://www.ncbi.nlm.nih.gov/LocusLink/LocRpt.cgi?l=4316

MMP26

http://www.ncbi.nlm.nih.gov/LocusLink/LocRpt.cgi?l=56547

MT4-MMP

http://www.ncbi.nlm.nih.gov/LocusLink/LocRpt.cgi?l=4326

MT6-MMP

http://www.ncbi.nlm.nih.gov/LocusLink/LocRpt.cgi?l=64386

ADAM1

http://www.ncbi.nlm.nih.gov/LocusLink/LocRpt.cgi?l=8759

ADAM2

http://www.ncbi.nlm.nih.gov/LocusLink/LocRpt.cgi?l=2515

ADAM12

http://www.ncbi.nlm.nih.gov/LocusLink/LocRpt.cgi?l=8038

ADAM9

http://www.ncbi.nlm.nih.gov/LocusLink/LocRpt.cgi?l=8754

ADAM10

http://www.ncbi.nlm.nih.gov/LocusLink/LocRpt.cgi?l=102

ADAM17

http://www.ncbi.nlm.nih.gov/LocusLink/LocRpt.cgi?l=6868

$\operatorname{src}$

http://www.ncbi.nlm.nih.gov/LocusLink/LocRpt.cgi?l=6714

ADAM11

http://www.ncbi.nlm.nih.gov/LocusLink/LocRpt.cgi?l=4185

\section{ADAM28}

http://www.ncbi.nlm.nih.gov/LocusLink/LocRpt.cgi?l=10863

ADAM15

http://www.ncbi.nlm.nih.gov/LocusLink/LocRpt.cgi?l=8751

ADAM19

http://www.ncbi.nlm.nih.gov/LocusLink/LocRpt.cgi?l=8728

ADAM8

http://www.ncbi.nlm.nih.gov/LocusLink/LocRpt.cgi?l=101

ADAM23

http://www.ncbi.nlm.nih.gov/LocusLink/LocRpt.cgi?l=8745
TIMP2

http://www.ncbi.nlm.nih.gov/LocusLink/LocRpt.cgi?l=7077

MT1-MMP

http://www.ncbi.nlm.nih.gov/LocusLink/LocRpt.cgi?l=4323

MMP9

http://www.ncbi.nlm.nih.gov/LocusLink/LocRpt.cgi?l=4318

TIMP3

http://www.ncbi.nlm.nih.gov/LocusLink/LocRpt.cgi?l=7078

TIMP1

http://www.ncbi.nlm.nih.gov/LocusLink/LocRpt.cgi?l=7076

TIMP4

http://www.ncbi.nlm.nih.gov/LocusLink/LocRpt.cgi?l=7079

MMP11

http://www.ncbi.nlm.nih.gov/LocusLink/LocRpt.cgi?l=4320

MMP14

http://www.ncbi.nlm.nih.gov/LocusLink/LocRpt.cgi?l=4323

MMP3

http://www.ncbi.nlm.nih.gov/LocusLink/LocRpt.cgi?l=4314

MMP13

http://www.ncbi.nlm.nih.gov/LocusLink/LocRpt.cgi?l=4322

MMP12

http://www.ncbi.nlm.nih.gov/LocusLink/LocRpt.cgi?l=4321

uPAR

http://www.ncbi.nlm.nih.gov/LocusLink/LocRpt.cgi?l=5329

TAT

http://www.ncbi.nlm.nih.gov/LocusLink/LocRpt.cgi?l=6898

NOTCH (NOTCH1)

http://www.ncbi.nlm.nih.gov/LocusLink/LocRpt.cgi?l=4851

ephrins

http://www.ncbi.nlm.nih.gov/LocusLink/LocRpt.cgi?l=1942

netrin 1

http://www.ncbi.nlm.nih.gov/LocusLink/LocRpt.cgi?l=9423

DCC

http://www.ncbi.nlm.nih.gov/LocusLink/LocRpt.cgi?l=1630

MCP3

http://www.ncbi.nlm.nih.gov/LocusLink/LocRpt.cgi?l=6354

RANTES

http://www.ncbi.nlm.nih.gov/LocusLink/LocRpt.cgi?l=6352 


\section{Biographies}

$\mathrm{V}$. Wee Yong is a central nervous system (CNS) glial biologist and neuroimmunologist whose research has been influenced by two diseases of the CNS: multiple sclerosis and malignant gliomas. Several lines of his investigations have converged on metalloproteinases as mediators of tissue injury or as facilitators of repair. Yong favours the concept that the precise spatial and temporal expression of specific metalloproteinase members in particular cell types determines whether these proteases serve as friends or foes in the CNS.

Christopher Power is a clinical scientist, neurologist and neurovirologist, who has been investigating the consequences of viral infections of the central nervous system (CNS). His work has implicated matrix metalloproteinases (MMPs) as the mediators of toxicity in the CNS after infections by several viruses, including HIV. The focus of his laboratory is directed towards understanding the mechanisms by which lentiviruses (HIV and FIV) cause neuronal death through the actions of MMPs.

Peter Forsyth is a clinical scientist and neuro-oncologist, who has investigated the aberrant expression of matrix metalloproteinases and tissue inhibitors of metalloproteinases (TIMPs) in facilitating the growth and invasiveness of malignant glioma cells. He has used TIMPs and other metalloproteinase inhibitors in pre-clinical testing in animal models of gliomas. Forsyth has also participated in clinical trials of metalloproteinase inhibitors in gliomas.

Dylan Edwards is interested in the roles of matrix metalloproteinases and ADAMs (a disintegrin and metalloproteinase) in normal and pathological tissue remodelling. He focuses on metalloproteinases in angiogenesis and cancer biology, as well as the gene regulation of metalloproteinases and tissue inhibitors of metalloproteinases.

\section{At a glance}

- Matrix metalloproteinases (MMPs) and ADAMs (a disintegrin and metalloproteinase) are part of a larger family of structurally related zinc-dependent metalloproteinases called metzincins. Structurally, MMPs are divided in three domains: an amino-terminal propeptide region, an amino-terminal catalytic domain, and a carboxy-terminal domain that is involved in substrate binding. ADAMs have a prodomain, a metalloprotease region, a disintegrin domain for adhesion, a cysteine-rich region, epidermal-growth-factor repeats, a transmembrane module and a cytoplasmic tail.

- The activity of MMPs is tightly regulated in several ways: at the level of transcription, by post-translational modifications such as proteolysis, and through the action of endogenous tissue inhibitors of metalloproteinases. The regulation of ADAMs is less well understood, although there is some evidence that the same three levels of regulation might control ADAM activity.

- MMPs and ADAMs have been implicated in neuroinflammation and multiple sclerosis (MS), in the pathogenesis of malignant gliomas, and in other neurological conditions such as stroke, viral infections and Alzheimer's disease. In the case of ADAMs, their role in these pathological states has begun to be explored, but the available literature is still in its infancy.

- Although the detrimental roles of metalloproteinases are well documented, some of their functions in the central nervous system (CNS) might be beneficial. For example, some metalloproteinases are expressed in the CNS during development, pointing to a possible role in brain maturation. Similarly, metalloproteinases have been implicated in myelinogenesis and axon growth. Furthermore, metalloproteinases are upregulated after injury to the CNS, indicating a possible relevance to tissue repair.

- Several challenges remain in the study of metalloproteinases and their role in brain function. It will be necessary to understand the balance between the beneficial and detrimental roles of MMPs to determine whether they can be used as targets for therapeutic intervention. It will also be important to identify the physiological substrates of the different metalloproteinases, and to develop selective antagonists against the various members of the metalloproteinase families; the lack of such tools constitutes one of the main limitations to the growth of the field at present. 